\title{
Standardless Quantitative EPMA of Microparticles with Irregular Shapes
}

Mouad Essani $^{1}$, Emmanuelle Brackx ${ }^{1}$, Philippe Jonnard ${ }^{2}$ and Emmanuel Excoffier ${ }^{3}$

${ }^{1}$ The French Alternative Energies and Atomic Energy Commission, Bagnols sur Cèze, LanguedocRoussillon, France, ${ }^{2}$ CNRS, Paris, Ile-de-France, France, ${ }^{3}$ The French Alternative Energies and Atomic Energy Commission, Bagnols sur Cèze, Ile-de-France, France

\section{Introduction}

Quantitative electron probe microanalysis (EPMA) is usually performed using analysis based on standards in which matrix effects are corrected by conventional ZAF or $\varphi(\rho z)$ models [1,2]. However, standard based models are developed for bulk specimens with plane surfaces and involve many assumptions about the thickness and morphology of the sample. These models are no longer accurate when quantifying the composition of microparticles. In fact, the generation and the emission of X-rays inside the particle can be significantly different than in a bulk polished sample. Moreover, analysis based on bulk standards does not take into account the loss of X-ray signal caused by the transmission of electrons through the microparticle.

Quantitative standardless procedures where X-ray intensities are calculated and used as virtual standards enable to overcome these limitations [3]. Standardless models based on Monte Carlo (MC) simulation were shown to be the most viable approaches to deal with nonconventional samples such as porous media [4], thin films [5] and microparticles [6]. Accurate X-ray intensity calculation can be obtained after including the geometry of the particle in the simulation. However, in some cases, particles can have very complicated shapes that would take a tremendous time and effort to describe accurately. Moreover, particles such as those used in the nuclear industry, often exhibit surface roughness which adds more complexities in the construction of their geometry. If not taken into account, irregularities in the particle's surface can be responsible for a loss in the measured X-ray intensities and might lead to multiple uncertainties in the quantitative results. In order to cope with this difficulties, peak-to-background (P/B) correction methods can be used to correct for the intensity loss due to surface roughness [8]. Standardless approaches based on P/B method were reported in a number of studies and showed that they are suitable for the quantification of micro-sized particles [9][10].

\section{Methods}

In this work, we present a P/B quantitative standardless approach in combination with MC simulation. Weight fractions are computed by means of the k-ratio between simulated and experimental P/B ratios. The virtual X-ray intensities are calculated using the general-purpose MC code PENELOPE [7]. For particles with usual geometries the form of the particle was constructed using the geometrical package "PENGEOM" of PENELOPE whereas for particles with undefined geometry, size effects were corrected after taking into account the reduction in the X-ray depth range caused by the transmission of electrons. A model was therefore developed to estimate the X-ray intensity loss by means of the ratio between the $\mathrm{X}$-ray generation range inside a particle and inside a bulk at a given electron energy. 


\section{Results}

In order to assess to validity of the approach, a study was performed on a calibrated NIST (National institute of Standards and Technologie) spherical microparticles of K411 glass. The analysis was further applied on $\mathrm{U}_{3} \mathrm{O}_{8}$ particles with undefined geometry. The correction of intensity loss due to size effects was achieved, which lead to promising quantitative results (figure 1). The chemical phase of $\mathrm{U}_{3} \mathrm{O}_{8}$ particles was afterward investigated using X-ray diffraction (figure 2) which enabled to validate the quantitative results obtained by EPMA.

\section{Conclusions}

Accurate quantification of small sized particles was achieved using a standardless procedure combining $\mathrm{MC}$ simulation and $\mathrm{P} / \mathrm{B}$ correction method. The approach offers the possibility to perform quantitative analysis of microparticles with complicated shapes and can be applied on particles assemblies that exhibit surface roughness with undefined geometries.

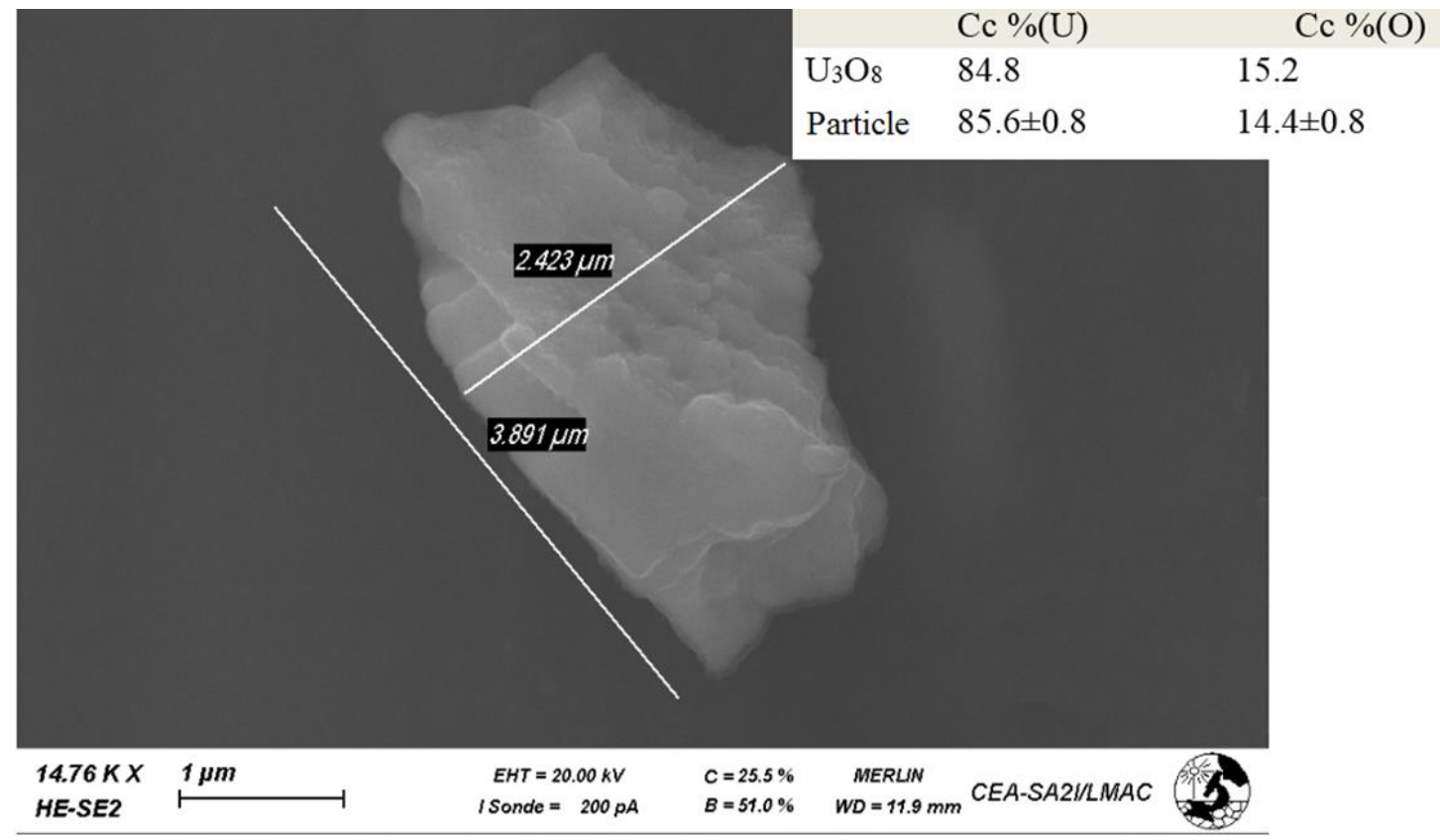

Figure 1. Example of an U3O8 microparticle with an irregular shape and its corresponding calculated elemental concentrations $\mathrm{Cc}$. The relative deviations from the elemental composition of a stoichiometric $\mathrm{U} 3 \mathrm{O} 8$ compound do not exceed $5 \%$. 


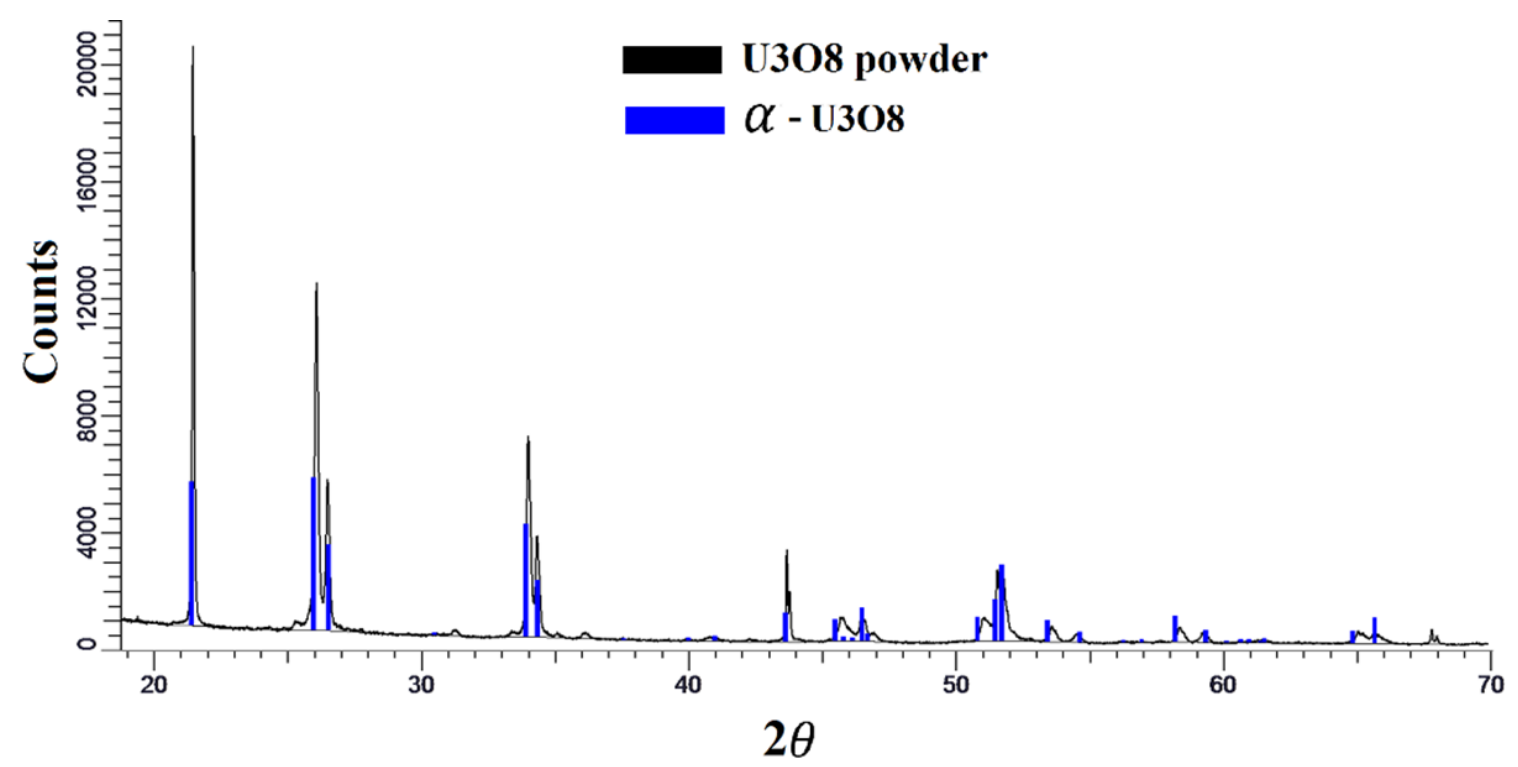

Figure 2. The measured X-ray diffraction spectrum of the studied U3O8 powder. The obtained crystalline structure agrees with the orthorhombic structure U3O8 of a stoichiometric U3O8 compound11.

\section{References}

(1) Philibert J. (1965) L'analyse quantitative en microanalyse par sonde électronique. Inst. De Recherches de la Sidérurgie, Saint-Germain-en Laye, France.

(2) Pouchou J.L.; Pichoir F. (1991). Quantitative Analysis of Homogeneous or Stratified Microvolumes Applying the Model "PA, In: Heinrich, K.F.J. and Newbury, D.E., Eds., Electron Probe Quantification, Plenum Press, New York, 31-75.

(3) Trincavelli J; Limandri S \& Bonetto R (2014). Spectrochim Acta, Part B, 101, 76-85.

(4) Sorbier L; Rosenberg E; Merlet C \& Xavier Llovet (2000). Mikrochim. Acta 132, 189-199.

(5) Hu Y \& PanY (2001). X-Ray Spectrom. 30: 110-115.

(6) Ritchie N.W.M (2010). Microsc. Microanal 16, 248-258.

(7) Salvat F; Fernández-Varea J. M. \& Sempau J (2011) J. PENELOPE-2011: A Code System for Monte Carlo Simulation of Electron and Photon Transport; OECD/NEA Data Bank, Issy-les-Moulineaux, France.

(8) Newburry D E (2004), Scanning vol. 26, 103-114

(9) Lábár J, Török S (1992). X-Ray Spectrom. 21 183-190

(10) Trincavelli J, Grieken R,V (1994). X-ray Spectrom 23, 254-260

(11) Taylor P, Wood D, Duclos A (1992). J. Nucl. Mater 189, 116-123 